\title{
Nuovo realismo e metodi di ricerca misti
}

\author{
Alessandra La Marca \\ Università degli Studi di Palermo, Dipartimento di Psicologia \\ doi: 10.7358/ecps-2014-009-lama \\ alessandra.lamarca@unipa.it
}

\section{NEW REALISM AND MIXED RESEARCH METHODS}

\begin{abstract}
"New Realism" has highlighted the limits of social constructivism and of so-called "weak thought» that risks producing nihilism. Some prejudices have recently been removed that prevented researchers and theorists from grasping the real core of educational facts in their concrete evidence. However, an opening to a metaphysical view would offer "new realism» further epistemological perspectives. In this sense, the contribution of critical realism might help to overcome the artificial antinomy between the active role of the knowing subject and the objective measurement of educational facts. With this «opening», scientific knowledge and current knowledge would no longer be seen as opposites because the latter would be considered as a deepening of the former, in a line of continuity. The experimental methods of research in the education field have recently made use of the philosophical reflections of realists to integrate the quantitative and qualitative approach to the study of education. New research designs, aimed at monitoring and observing educational processes, without "encaging" them, are mainly of a "mixed» kind.
\end{abstract}

Keywords: Critical realism, Experimental method, Qualitative approach, Quantitative approach, Research designs.

Dopo decenni in cui il "pensiero debole» ha goduto di diffuso ascolto, da qualche tempo si mostrano segnali di un diverso orientamento all'insegna di un «nuovo realismo» (Ferraris, 2012).

Il nuovo realismo si presenta come erede del postmoderno. Se il postmoderno ha avuto il merito di dire addio alle grandi narrazioni e di svelare i meccanismi di potere che governano la società, ha tuttavia anche virato deci- 
samente verso un relativismo spinto, che si è tradotto in nichilismo, laddove la celebre frase nietzscheiana «non ci sono fatti, solo interpretazioni» è stata intesa fino a dissolvere la realtà. Il nesso oggettività/evidenza è indubbiamente al centro di dibattiti tesi e controversi.

Concordo con Mari (2013, p. 197) nell'affermare che il tratto di maggiore interesse del nuovo realismo - dal punto di vista pedagogico oltre che da quello filosofico - risieda nella lucida critica al "pensiero debole» e all'ermeneutica da questo influenzata che ha avuto ricadute anche per quanto concerne la teoria dell'educazione.

Rivalutare la possibilità di riconoscere il reale significa quindi rivalutare un'ontologia seppur minima, il fatto che «il mondo ha le sue leggi e le fa rispettare».

Non si può fare a meno del reale, del suo starci di fronte; non si può essere indisponibili a negoziare. Sia quello che sia, ci renda felici o infelici, il reale è qualcosa che resiste e che insiste, ora e sempre, come un fatto che non sopporta di essere ridotto a interpretazione.

Abbiamo bisogno non solo di conoscere, ma di alcune verità stabili che non mutino ad ogni vento, e questo è antinichilismo, essendo la negazione del sapere e della verità un volto fondamentale del nichilismo. Quello di cui ora si avverte la necessità non è tanto una nuova teoria della realtà (né meno che mai una "teoria della nuova realtà», che suona minacciosa anche solo a leggerla), quanto piuttosto un lavoro che sappia distinguere, con pazienza e caso per caso, che cosa è naturale e cosa è culturale, che cosa è costruito e cosa no. È questo, soprattutto, il senso di una grande trasformazione che - a livello mondiale - ha investito la filosofia, portandola fuori dai vicoli ciechi che nel secolo scorso hanno indotto molti a parlare della sua fine.

In cosa consiste la verità? In cosa consiste la realtà? Come stabilire il loro statuto, la loro ricerca, la loro posizione? Quali gli ambiti di applicazione in cui, oggi, questi due concetti chiave della storia del pensiero possono e devono essere recuperati? Che cosa conosciamo quando conosciamo? La realtà è socialmente costruita e infinitamente manipolabile?

Il nuovo realismo cerca faticosamente una risposta a tali domande ma quando perde il riconoscimento dell'esistenza della realtà in ottica metafisica sfocia in derive di tipo fenomenico-materialista.

Il nuovo realismo reagisce opportunamente a postmodernismo e debolismo e sembra voler rilanciare la sfida col reale nella semplicità di uno schema che riduce gli oggetti in tre classi: gli oggetti naturali, esistenti nello spazio e nel tempo indipendentemente dai soggetti; gli oggetti sociali, la cui esistenza nello spazio e nel tempo dipende invece dai soggetti stessi; e gli oggetti ideali, che esistono fuori dello spazio e del tempo indipendentemente dai soggetti. Lorientamento del nuovo realismo pur essendo di grande interesse, presenta 
però un problema di fondo, ben descritto da Mari (2013): il rischio della contrazione della realtà a costrutto puramente descrittivo.

Per il «pensiero debole», ricorda Ferraris, la realtà non è mai accessibile in quanto tale, visto che è mediata dai nostri pensieri e dai nostri sensi, e per decenni appellarsi ad essa è stato ritenuto filosoficamente vacuo. Questo, con mille sfumature, è stato ed è tuttora il clima del pensiero postmoderno in Italia, simboleggiato da figure come Gianni Vattimo e Pier Aldo Rovatti, che hanno legato il loro nome, appunto, al cosiddetto «pensiero debole».

Possenti (2013) ${ }^{1}$ pur riconoscendo a Ferraris il merito di un risveglio dal «sonno» del postmoderno, mette in luce il respiro corto del nuovo realismo. La fragilità della proposta neo-realista è messa in luce in modo serrato anche da D'Agostini (2013), nelle sue contraddizioni interne che derivano, come per gran parte della speculazione postmoderna, dall'aver dimenticato i parametri logici di quella che Aristotele chiamava "filosofia prima».

La sfida del reale non ammette riduzioni. Fondare la verità su una determinata visione degli oggetti deriva dalla mancata assunzione della storicità delle possibilità che a tale oggetto ineriscono. Possibilità che non sono infinitamente manipolabili, come l'estetizzazione postmoderna può farci credere, perché si radicano nell'identità di ciascuno, che è in relazione con lo spazio comunitario e con l'orizzonte di senso a cui è legato.

Il rischio di un tale tentativo per M. De Caro e M. Ferraris (2012) ${ }^{2}$ è quello di apparire una ripresa ingenua del vecchio realismo. Non si tratta di tornare al realismo ingenuo, che accetta acriticamente le realtà basate sulle pratiche quotidiane. "La grande sfida che si presenta al realismo filosofico dei prossimi anni è dunque quella di conciliare le componenti positive del realismo del senso comune e del realismo scientifico, depurandole dalle rispettive componenti negative in modo da concepire una realtà inclusiva in cui esistano veramente tanto il mondo ordinario quanto quello della microfisica» (De Caro, 2012, p. 38).

1 Possenti si considera un «nativo realista», termine che vuole significare qualcosa di simile al termine «nativo digitale». Mentre questi ultimi esistono da poco, da lungo tempo vi sono i «nativi realisti». L'istanza realistica è assolutamente decisiva nell'interpretazione di uno dei massimi problemi che da quasi due secoli assillano l'Occidente: la questione del nichilismo ed in specie di quello teoretico, cui Possenti ha dedicato vari lavori ed in particolare, Nichilismo e metafisica. Terza navigazione (Roma: Armando, 2004). Per questo filosofo il nichilismo non può essere inteso adeguatamente se non come esplicito antirealismo, oblio dell'essere e distruzione dell'idea di verità.

2 Il volume dà conto del dibattito filosofico iniziato proprio su Repubblica nell'agosto del 2011, che aveva come punto chiave l'idea di superare il postmoderno. Da allora molti sono stati i contributi, favorevoli e contrari, a un ritorno alla «realtà». In questo libro vengono raccolti i saggi di vari nomi celebri, da Eco a Putnam, che mostrano come la posizione realista possa avere oggi più di una sfumatura. 
Nell'agosto del 2011, con la pubblicazione sulle pagine di Repubblica del «Manifesto del nuovo realismo», Maurizio Ferraris ha smosso le acque del dibattito filosofico.

Ferraris (2011, pp. 59 e 70) pone esplicitamente in antitesi il nuovo realismo con il positivismo e con la concezione strumentalistica della scienza: «[...] contro il positivismo che esalta la scienza e contro il postmoderno che la riduce a una lotta fra interessi», egli propone "un rilancio della filosofia come ponte tra il mondo del senso comune, dei valori morali e delle opinioni e il mondo del sapere in generale».

Il nuovo realismo è anzitutto la presa d'atto di un cambio di stagione. L'esperienza storica dei populismi mediatici, delle guerre "post 11 settembre» e della recente crisi economica ha portato una pesantissima smentita di due dogmi centrali del postmoderno: l'idea che la realtà sia socialmente costruita e infinitamente manipolabile, e che la verità e l'oggettività siano nozioni inutili. Le necessità reali, le vite e le morti reali, che non sopportano di essere ridotte a interpretazioni, sono tornate a far valere i loro diritti.

Il nuovo realismo si propone proprio di conservare le istanze postmoderne, evitandone però l'esito ultimo, laddove dichiara guerra alla realtà poiché non solo una Verità, ma anche le molteplici verità (con la «V» minuscola) sono considerate inesistenti. Per dirla con Ferraris (2011, p. 9) "[è] sacrosanto decostruire: in natura non esistono i granduchi, i padri-padroni e gli angeli del focolare, essi sono socialmente costruiti. Ma questo non significa che tutto sia socialmente costruito".

Come afferma De Caro $(2013, \ldots)$, per comprendere la cruciale rilevanza filosofica del problema del realismo, la prima cosa da notare è che, nonostante ciò che talora si legge, tale problema non ha la forma "tutto o niente». Detto in altro modo, mai nessun filosofo è stato del tutto realista e mai nessuno del tutto antirealista. Il problema sta fondalmentalmente nel determinare quale sia la giusta dose di realismo da adottare. E questo non è certo un compito semplice o irrilevante.

In dialogo critico con Vattimo, Ferraris ha notato che il primato delle interpretazioni sopra i fatti e il superamento del mito della oggettività, cui si richiamavano i debolisti, non hanno avuto gli esiti di emancipazione che immaginavano filosofi postmoderni come Richard Rorty (2012) ${ }^{3}$.

3 Nel Manifesto pubblicato su Repubblica Maurizio Ferraris afferma di voler «restituire lo spazio che si merita, in filosofia, in politica e nella vita quotidiana, a una nozione, quella di «realismo", che nel mondo postmoderno è stata considerata una ingenuità filosofica e una manifestazione di conservatorismo politico». 


\section{ATTUALITÀ DEL «REALISMO CRITICO» di J. Maritain, E. Gilson e G. Corallo}

La recente proposta del nuovo realismo induce a riconsiderare le posizioni di tre pensatori del XX secolo che, secondo me, offrono fecondi spunti per superare i limiti del costruttivismo sociale senza negare il ruolo attivo svolto dal soggetto nel far emergere quanto di vero c'è nella realtà conoscibile.

Il neotomismo, che deve molto anche ad Aristotele, fa del riconoscimento della "originarietà» della realtà un punto imprescindibile, relativizzando la conoscenza scientifica e avvalorando un pensiero che non disprezza i dati, come mostra la coltivazione della psicologia e della pedagogia sperimentali nelle università cattoliche di Lovanio, Friburgo e Milano, altrettanti centri di irradiazione del neotomismo pedagogico (Mari, 2013, p. 195).

La metafisica aristotelica, a cui si sono rifatti Gilson, Maritain e Corallo, esprime una singolare capacità di unificare le conoscenze senza uniformarle, costituendo un paradigma teoretico particolarmente fruibile per quanto concerne le scienze dell'educazione.

La ricerca dell'unità del sapere costituisce una meta molto esigente e all'altezza dell'attuale momento storico. Per salvare l'uomo vale la pena rompere schemi rutinari ed isolanti nel tentativo di superare la frammentazione. La settorialità del sapere, in quanto comporta un approccio parziale alla verità con la conseguente frammentazione del suo significato, impedisce il conseguimento di quell'unità interiore alla quale ogni uomo aspira. Con la frammentazione l'attività conoscitiva produce una molteplicità di dati e di conoscenze senza una visione unitaria del reale. L'uomo si trova oggi a dover agire in un mondo del quale ha soltanto delle immagini parziali e scollegate; da ciò nasce un senso d'insicurezza, che viene provvisoriamente nascosto dai risultati della tecnologia. Anche in questo caso si nota come tutte le scienze rimandano, non solo alle questioni di metodo scientifico, ma anche a temi di antropologia filosofica.

Nel libro Distinguere per unire. I gradi del sapere Jacques Maritain (anno della prima edizione) utilizzando il «metodo» del realismo critico raggiunge una sintesi teoretica dell'universo variegato delle teorie della conoscenza percorrendo un itinerario che si snoda attraverso le correnti principali del pensiero antico e moderno.

Il realismo classico-tomista, da cui muove il pensiero maritainiano, ha sempre tenuto insieme dottrina della conoscenza, ontologia e metafisica, a differenza del New Realism anglosassone il quale ha lasciato da parte sino ad un recente passato la domanda metafisica.

La filosofia dell'essere, soprattutto nell'interpretazione che del tomismo ha dato Maritain, offre una spiegazione del rapporto «intenzionale» con cui l'intelletto coglie l'ente, che resta insuperata (Possenti, 2012). 
Nel libro appena citato Maritain affronta in modo rigoroso le tesi gnoseologiche che si contrappongono alla sua concezione, come quelle del positivismo, del neopositivismo logico, del materialismo storico dialettico, dell'idealismo, della fenomenologia di tendenza husserliana-idealista

Maritain ritiene possibile raggiungere una conoscenza reale dell'essere e dell'esistenza, al seguito di una elaborazione accurata dei modi con cui la nostra mente si rapporta all'essere e lo conosce. Secondo Maritain, nel dare sostanza all'ineludibile ricerca sulle possibilità e i limiti della conoscenza del reale, non possiamo cavarcela ricorrendo esclusivamente alla logica, al principio di non contraddizione o a meri enti di ragione. Rimanere ostinatamente nell'ambito della logica conduce a teorizzare su ciò che è soltanto pensato; applicare poi tutto ciò all'essere reale ed esistenziale è rimanere vittime di una straordinaria allucinazione.

Una via di uscita proviene dal riprendere la riflessione sul rapporto originario tra senso, intelletto ed essere, pervenendo alla conclusione che nessuna interfaccia cognitiva dapprima conosciuta si frappone tra intelletto e oggetto: dunque né la nostra percezione sensibile né quella intellettuale sono tagliate fuori dall'esistenza, dall'essere reale. In fin dei conti, per Maritain, la nostra conoscenza può attingere il reale così come è, può raggiungere quell'atto di esistere (actus essendi) che, pulsando in ogni ente, costituisce la scaturigine primaria del suo proprio esistere.

Nel saggio Il realismo, metodo della filosofia Étienne Gilson (2008) propone di seguire una via antica, abbandonata da Cartesio e da tutti quegli autori che possono definirsi idealisti.

Secondo Gilson, l'idealismo tende ad «ipostatizzare» il proprio metodo (dell'immanenza), fino ad arrivare al paradosso per cui non è più questo che si adatta alla realtà, ma è la realtà stessa che «è forgiata» dal metodo in una forma di minor valore rispetto al suo concetto, e quindi, in un certo senso, svalutata, corrotta, illusoria. Questo perché l'idealismo - continua l'autore - non ha capito, o voluto capire, che la conoscenza non è il pensiero. Nel senso che il realismo si occupa della conoscenza in quanto concetto che va oltre il pensiero, perché per il realista "pensare» significa organizzare delle conoscenze che però sono «esterne» al pensiero, non provengono dal pensiero stesso, mentre l'idealismo considera la conoscenza come pensiero che riflette sul proprio contenuto. Per l'idealista, quindi, la "critica» della conoscenza consiste in un tentativo di fondare a priori le condizioni stesse del conoscere, una volta abbandonato il terreno del realismo. E non ha senso, allora, pensare ad un realismo che sia anche "critico», perché l'aggettivo non può applicarsi che ad una filosofia svuotata dell'oggetto della conoscenza, che allora non sarà più realista.

Gilson (2008, p. 196) così sintetizza il principio fondamentale del realismo: «[...] il fatto che ogni esistenza mi sia data in una conoscenza non 
implica assolutamente che la mia conoscenza sia la causa di questa esistenza. [...] Che tutto mi sia dato nel pensiero non permette minimamente di affermare che tutto si riduce al pensiero». Grazie all'«evidenza sensibile iniziale» (Gilson, 2008, p. 196), vi è anzitutto «'esperienza immediata di un in-sé, nella quale poi, attraverso uno sforzo di riflessione, l'io prende coscienza di sé» (ivi, p. 124).

La consapevolezza di stare pensando un oggetto che esiste indipendentemente da me, ma la cui verità non può esistere senza che io la pensi - tipica affermazione coralliana - consente di qualificare come realista la gnoseologia di Gino Corallo, che è aperta alla metafisica, intesa come fonte remota della pedagogia ${ }^{4}$.

La verità è, in un certo senso, sempre nuova: il suo apparire alla mente di chi la trova non dovrebbe mai avvenire senza quel brivido di emozione e di meraviglia che indica, con la commozione del soggetto, la sua attiva partecipazione, il suo attivo prendere parte alla costruzione della verità. [...] La curiosità intellettuale è la freschezza e la gioia della meraviglia, freschezza e gioia che nascono solo, come si è detto, dall'attiva partecipazione del soggetto alla situazione di «verità». Questo non significa che la verità sia riducibile tutta a un fatto soggettivo, o a una pura creazione da parte del soggetto, rispetto a una relazione situazionale. [...] Qui ci interessa sottolineare il fatto che la ricerca, o anche la trasmissione, della verità non possono avvenire senza che il soggetto, o i soggetti, in essa impegnati si pongano come attivi co-autori della verità stessa. Questa, si è detto, non perde così il suo carattere oggettivo, non diventa la «mia» verità, ma diventa - inconfutabilmente - una verità mia. (Corallo, 1966, pp. 26-27)

Possiamo dire con Corallo che l'uomo non inventa la realtà, nel senso che le cose non dipendono dalla nostra conoscenza di esse, ma è parimenti innegabile che il pensiero umano arricchisce la realtà. Risulta fondamentale tenere ferma la distinzione tra l'esistenza di un oggetto e l'esistenza della verità di quell'oggetto; nel secondo caso è necessario il lavoro dell'intelligenza umana. Per le sue conseguenze nel campo della ricerca pedagogica, Corallo evidenzia che il pensiero è lo strumento che l'uomo ha a disposizione per conoscere la realtà, per scoprire la verità che c'è nelle cose e non per inventarla né individualmente né socialmente. La preoccupazione del ricercatore sarà sempre

4 Corallo esplicita la sua metafisica nelle pagine 44-64 del primo volume del suo trattato di pedagogia. Si tratta di un modo originale di far evolvere la metafisica aristotelico-tomista nel dibattito con l'idealismo e il positivismo alla metà del XX secolo. Nell'ultimo scritto della sua carriera accademica, dove sintetizza il suo percorso di pensiero (è del 1985 ma fu pubblicato per la prima volta nel 1988), per qualificare in qualche modo la sua concezione filosofica usò l'espressione «sintesi trascendentale a simultaneo». 
quella di rispettare una serie di regole imposte dal pensiero logico al fine di salvaguardare la realtà e l' aderenza ai fenomeni studiati.

Corallo (1966, pp. 70-83) segnala che nel lavoro scientifico si opera un continuo trapasso fra il rilievo dei fatti, la formulazione di generalizzazioni (ipotesi) per spiegare i fatti, la deduzione delle conseguenze di queste ipotesi, la ricerca di nuovi fatti per accertare le ipotesi. Nel caso della deduzione il ricercatore muove da principi universali verso fenomeni particolari, mentre nel caso dell' induzione egli muove dal particolare verso l'universale. Solo l'uso logicamente corretto ed alternato del ragionamento induttivo e di quello deduttivo permette al ricercatore di arrivare a conclusioni valide. Il pedagogista siciliano ritiene arbitraria una netta distinzione tra il metodo induttivo e il metodo deduttivo perché di fatto c'è un unico ragionamento compiuto dal pensiero nel suo movimento, nel senso che l'induzione offre le basi per le ipotesi, mentre la deduzione indaga sulle conseguenze logiche delle ipotesi, allo scopo di eliminare quelle che sono in contrasto con i fatti; l'induzione poi contribuisce ancora alla verifica delle restanti ipotesi.

\section{Conoscenza Abituale E CONOSCENZA SCIENTIFICA}

Il realismo critico costituisce, fin dall'inizio, il quadro filosofico di riferimento della ricerca sperimentale di Luigi Calonghi (1957) in campo educativo. La ricerca è un modo di conoscere, di porsi in contatto con la realtà educativa, un modo rigoroso ${ }^{5}$, ma sostanzialmente non dissimile dalla forma corrente del conoscere umano. Le fasi dei due tipi di conoscenza - corrente e scientifica - sono essenzialmente le stesse, anche se è un po' diverso il modo d'interpretarle e di attuarle (Calonghi, 1980).

Qualunque attività conoscitiva, e quindi anche la conoscenza scientifi$\mathrm{ca}$, anzi forse questa più di altre, è una ricerca che impegna l'uomo in tutte le sue facoltà, si potrebbe dire in tutto il suo spirito. La conoscenza deve essere per forza qualcosa di proprio della persona, di personale; ma allo stesso tempo è oggettiva, perché è suscitata quasi dalla realtà stessa, è ancorata a essa e quindi diventa verificabile nelle diverse forme in cui si esprime.

La differenza fondamentale tra conoscenza abituale e conoscenza scientifica può essere ricondotta al fatto che in quest'ultima si ha una maggiore razionalità nei controlli e nei procedimenti. In pratica la conoscenza scientifica - in questo caso specifico, la ricerca pedagogica di tipo sperimentale - ci

5 Per i rapporti tra esperienza, innovazione, aggiornamento e sperimentazione si può vedere Calonghi, 1973. 
appare come un potenziamento, un miglioramento del nostro modo abituale di conoscere i fatti educativi.

L'osservazione, nella conoscenza abituale, non è sistematica e si identifica in molti casi con una "percezione» intuitiva, episodica, della situazione, senza particolari preoccupazioni di razionalità, di obiettività, di completezza rispetto a criteri prescelti. Nel conoscere scientifico l'osservazione viene invece condotta secondo norme rigorose che garantiscono il massimo rispetto e la massima penetrazione della realtà; la fase di osservazione, nell'ambito di una ricerca positivo - sperimentale, include anche il periodo in cui si fa maturare un'ipotesi attraverso tentativi e progressi successivi.

L'ipotizzare, continua Calonghi, è un modo rigoroso, sotto l'aspetto logico, di formulare una supposizione. Nell'ambito della conoscenza scientifica si esige che l'ipotesi sia formulata in modo da mettere in evidenza conseguenze specifiche, verificabili sperimentalmente. Tali conseguenze devono essere derivate secondo le regole della deduzione, rigorosamente osservate. L'aspettativa che caratterizza il conoscere abituale risponde implicitamente e sommariamente a tali regole, e si traduce in termini solitamente meno specifici.

La verifica nella conoscenza abituale può essere sommaria, globale, affidata più o meno a stime intuitive. Nella conoscenza scientifica, invece, deve consentire la conferma di rapporti costanti tra un comportamento osservato e dei fattori ipotizzati; una conferma tanto sicura da render possibile predizioni attendibili, con limitate e precise probabilità d'errore.

Dunque, sostiene Calonghi, sia lo scienziato sia l'uomo comune, per la produzione di conoscenza, raccolgono informazioni (garantite da evidenza empirica) per trovare la soluzione a un determinato problema, o meglio, la risposta a una precisa domanda per la quale non ritengono di possederne già una accettabile. La principale differenza fra i due modi di procedere, quello della conoscenza scientifica e quello guidato dalla saggezza "quotidiana», risiede essenzialmente nel fatto che nel primo dei modi le procedure e le scelte adottate devono essere rese esplicite e sistematiche. Pertanto la ricerca scientifica può essere sinteticamente definita come «un processo di osservazione deliberata e controllata». Di conseguenza, anche la valutazione dei risultati assume, nella conoscenza scientifica, un valore di generalizzabilità che la conoscenza abituale non potrebbe consentire. Sia nella ricerca scientifica che nell'indagine empirica è il problema a guidare l'attività conoscitiva della persona che vuole raggiungere alcuni obiettivi. La conoscenza scientifica presenta però delle caratteristiche che non si trovano nella conoscenza spontanea, per quanto attiene all'osservazione, alla formulazione delle ipotesi e alla loro verifica.

Calonghi ricorda ancora che prima di raccogliere informazioni utili per elaborare la risposta a una domanda bisogna formulare in modo esplicito il 
problema, ovvero si devono individuare i diversi fattori (dimensioni, variabili) potenzialmente rilevanti rispetto al problema stesso e avanzare delle ipotesi circa gli effetti semplici o congiunti di tali fattori. Nel fare questo il ricercatore utilizza (o elabora) una teoria di riferimento ed è sulla base di essa e delle proprie intuizioni che egli avanza delle ipotesi di possibili soluzioni. Qualunque indagine scientifica esige, infatti, una teoria (sia essa di tipo esplicito o implicito) che consiste in un insieme interrelato di concetti, di definizioni e proposizioni in grado di fornire una visione sistematica dei fenomeni specificando le relazioni tra le variabili, al fine di spiegare e prevedere i fenomeni stessi. Mentre nella conoscenza basata sul buon senso ciò non è richiesto, la conoscenza scientifica esige la scelta di un metodo rigoroso per elaborare una teoria basata sull'evidenza dei dati raccolti.

Pur non esistendo un metodo privilegiato per giungere alla elaborazione di una teoria, tuttavia i diversi metodi devono rispondere a certi requisiti per poter essere definiti «scientifici». Calonghi ne elenca tre: la riproducibilità, l'organicità e la verificabilità.

a. Innanzitutto un metodo scientifico deve essere oggettivo: conducendo le sue osservazioni attraverso un empirismo stretto e ragionando con la logica formale, il ricercatore cerca di assicurare oggettività al suo approccio. Di conseguenza, se il ricercatore giunge a una conclusione basata su una certa evidenza, altri ricercatori devono poter raggiungere la stessa evidenza e arrivare alla stessa conclusione o a una equivalente (principio della riproducibilità).

b. Poiché gli obiettivi a lungo termine dell'approccio scientifico sono la teoria e la scienza, una metodologia di ricerca è scientifica nella misura in cui contribuisce all'elaborazione teorica. Ulteriore caratteristica di una metodologia scientifica è pertanto l'organicità. Lo scienziato generalmente non è interessato a semplici frammenti di informazione ma a un quadro complessivo di conoscenze; una buona metodologia scientifica cerca quindi di apportare contributi significativi al processo di scoperta di leggi o principi generali che governano determinati aspetti della realtà. Si considera conoscenza generale e sistematica quella scienza costituita da un insieme interconnesso di teorie e proposizioni specifiche dedotte da principi generali. Data questa interrelazione possiamo dire, ad esempio, che - anche nel caso ci fosse la «scienza delle comunicazioni di massa» - non per questo esisterebbe un'unica teoria delle comunicazioni di massa.

c. Infine, chi si occupa di metodologia di ricerca, come di ogni altra disciplina, deve essere consapevole che tutte le conoscenze sono suscettibili di cambiamenti e miglioramenti: dubbi e interrogativi meritano quindi la massima attenzione. Leggi e principi devono essere soggetti a una costante valutazione critica. In altri termini, il ricercatore è chiamato a sottopor- 
re sempre il suo lavoro all'esame critico degli altri ricercatori che operano nello stesso campo. La terza caratteristica pertanto di una metodologia di ricerca è la sua verificabilità da parte della comunità scientifica.

Il rispetto dei principi che assicurano il rigore metodologico non esclude tuttavia che un ricercatore possa condurre degli studi esplorativi per soddisfare la sua curiosità e il suo desiderio di una migliore comprensione di situazioni ed eventi oppure per verificare la possibilità di fare studi più approfonditi o, infine, per sviluppare gli strumenti necessari per una successiva ricerca rigorosa.

\section{LA PARTECIPAZIONE PERSONALE NELLA CONOSCENZA OGGETTIVA DEL REALE}

L'impossibilità di giungere alla conoscenza oggettiva della realtà educativa è motivata a volte dalla considerazione che il ricercatore non riesce ad essere neutrale. A parte il fatto che è sempre meglio avvicinarsi il più possibile a cogliere realtà per come essa è piuttosto che rinunciare in partenza a sapere, sia pure imperfettamente, qualcosa in più su un fatto educativo, conviene ribadire che la conoscenza assume il carattere della scientificità non quando pretende di essere del tutto oggettiva ma quando il ricercatore è consapevole del suo valore e dei suoi limiti grazie al controllo operato sulle procedure di ricerca e su stesso. La conoscenza personale non è, in quanto personale, «un atto arbitrario [e che dunque ci può essere o non essere a seconda delle decisioni prese] né un'esperienza passiva, ma un atto responsabile che aspira alla validità universale» (Polanyi, 1990, p. 70); al contrario, «l'ideale di una conoscenza che consiste in affermazioni strettamente impersonali ci appare come contraddittoria e senza significato, al limite del ridicolo. Dobbiamo imparare ad accettare come nostro ideale una conoscenza che sia chiaramente personale» (Polaniy, 1959).

L'interazione fra soggetto conoscente e realtà conosciuta è determinante tanto per la dinamica di ogni processo oggettivante, quanto per lo sviluppo del progresso scientifico. Contrariamente a quanto si possa a prima vista pensare, questa prospettiva non ha nulla del soggettivismo relativista, né dell'a priori categoriale del razionalismo kantiano, né si ricollega all'indeterminismo fenomenico che compare in alcuni aspetti delle scienze sperimentali. La presenza nel soggetto di una certa visione del mondo in ogni approccio alla natura, visione che nasce dal reale ed al reale conduce, e che nell'analisi delle scienze viene identificata con il ruolo della conoscenza intuitiva in quanto forgiata dall'esperienza, è ciò che Polanyi chiama «conoscenza 
tacita» (Vinti, 1999). Si sottolinea allo stesso tempo che ogni conoscenza non è isolatamente oggettiva, ma anche soggettiva, perché data in un soggetto conoscente e, pertanto, radicalmente personale.

Come deve agire lo studioso nell'attuare la «conoscenza personale»? Risponde Torrance in Belief in science and in Christian life (1980, p. 15)

Secondo Polanyi qualsiasi ricerca scientifica portata avanti in un modo distaccato, impersonale e materialista isola se stessa dalle facoltà più alte dell'uomo e dunque restringe il suo campo e il suo potere di discernimento e comprensione. L'autore aggiunge che l'obiettivo dello scienziato è quello di trovare «un nuovo approccio in cui superare la dannosa cesura tra soggetto ed oggetto, mente e materia, o pensiero ed esperienza, e ricostituire la naturale unità del conoscere e dell'essere, poiché senza il modo integrativo di pensare che tale equilibrio razionale porta, la scienza può solo ostacolare i suoi stessi tentativi di cogliere le strutture più fini e più delicate incastonate nella natura» (ivi, p. 15).

In tutti gli atti di comprensione vi è dunque una partecipazione personale determinante che non rende però l'attività conoscitiva un atto arbitrario o un'esperienza passiva; ma, tutt'altro, la qualifica come atto responsabile, che non può in nessun caso essere neutro o scevro da supposizioni e implicazioni contestuali, ma che nello stesso tempo aspira alla validità universale. Si realizza così un conoscere che è oggettivo nel senso che stabilisce un contatto con una realtà nascosta che si crede conoscibile e reale.

L'oggettività si pone sempre come appello alla razionalità del soggetto e nel soggetto, come un richiamo dell'essere che non può tradursi se non in una opzione per il vero; tale opzione coinvolge la persona in un rapporto dialogico impegnativo con la realtà. Questa partecipazione personale del ricercatore alla conoscenza della realtà non contrasta neppure con il realismo radicale di Searle (2012), per il quale il presupposto di qualsiasi filosofia «sana», è l'accettazione della realtà come dato imprescindibile di ogni nostro pensiero sul mondo. La nostra esperienza quotidiana è possibile proprio perché c'è un mondo che esiste indipendentemente dalle nostre sensazioni e fantasie. Se non ci fosse questo mondo di sostanze - alberi, montagne, spiagge, esseri umani e altri corpi - gran parte delle nostre azioni sarebbero prive di senso. Searle mostra di prendere molto sul serio l'ontologia, cioè lo studio dei vari sensi in cui possiamo dire che ci sono delle cose ${ }^{6}$.

Si pensi alla risolutezza di Searle quando parla del caso allucinatorio. Alla domanda: «[...] se io percepisco una sedia, come posso sapere se questa sedia c'è davvero o è solo nella mia mente?». Il filosofo americano dà la stessa

6 John Searle riprende nel suo libro Mente, linguaggio e società, i temi trattati nell'arco di oltre trent'anni (gli atti linguistici, l’intenzionalità, la coscienza e la società) cercando di «salire dai livelli della mente e del cervello al linguaggio e alla realtà sociale in generale». 
risposta che probabilmente avrebbero dato le nostre nonne. Se ti sembra che ci sia una sedia ma la sedia non c'è, ti stai semplicemente sbagliando.

Nella vita mentale di un uomo, afferma Searle (2012) i limiti della ragione sono, per così dire, già inclusi negli stessi strumenti conoscitivi che la natura ci ha offerto: la percezione, il pensiero, il ragionamento. Proprio perché tali limiti vincolano la nostra condizione di esseri intenzionali, essi svolgono un ruolo speciale anche riguardo ai temi dell'etica, a quei rapporti tra persone che chiamano in causa questioni di vitale importanza.

Contro il vecchio scetticismo che, ancora nel XVII secolo, sollevava dubbi sulla realtà, Searle contrappone le odierne conquiste scientifiche e tecnologiche. Contro il dualismo tra realtà fisica e realtà mentale Searle contrappone poi la sua visione della realtà come una, della quale la mente fa parte. Non accetta quindi - non può accettare - neppure la posizione secondo la quale non possiamo percepire il mondo reale, ma solo ciò che accade nella nostra mente.

\section{RICERCA QUANTITATIVA E RICERCA QUALITATIVA: UNA NECESSARIA INTEGRAZIONE}

La ricerca empirica in campo educativo si è recentemente avvalsa delle riflessioni filosofiche dei realisti per integrare l'approccio qualitativo e quello quantitativo nello studio dei fatti educativi.

Quanto è stato appena detto sulla relazione tra l'ineliminabile soggettività del ricercatore e l'esigenza di avvicinarsi il più possibile alla realtà oggettiva con la ricerca scientifica, consente di superare l'antica querelle tra il "quantitativo» e il "qualitativo». Ricomponendo il quadro sulla base di un insieme di assunti che sembrano accomunare oggi tutti i ricercatori, non è difficile constatare come si pongano le basi per superare le classiche distinzioni tra ricerca qualitativa e quantitativa.

Oggi il dibattito in corso a livello internazionale riguarda il cosiddetto approccio eclettico, la prospettiva che accetta qualunque combinazione di metodi purché si giunga a qualcosa di significativo (Pellerey, 2011, p. 110).

È oggi condivisa tra i ricercatori l'idea che triangolando i risultati ottenuti con metodi quantitativi e qualitativi, sia possibile valutare la consistenza o la stabilità delle conclusioni di una ricerca, tenendo tuttavia presente che la realtà educativa è delicata, complessa e difficile da cogliere; quindi chi si dedica a studiarla deve avere la chiara consapevolezza che nella conoscenza di qualunque fatto educativo reale rimane sempre un margine di incertezza (Garcia Hoz, 1997 e 1988). 
Trinchero (2004) ricorda che, nella ricerca, oggi il fuoco non è più sul metodo - approccio troppo legato al dualismo qualitativo/quantitativo - ma sulle strategie di ricerca, che vanno stabilite sulla base di un'accurata definizione dei propri obiettivi conoscitivi e del contesto in esame, mantenendo una visione il più possibile aperta e multi-metodologica, tanto in situazioni che richiedono ricerca nomotetica - nel caso l'obiettivo sia quello di astrarre dalla situazione oggetto di studio leggi e regole più generali -, quanto in situazioni che richiedono il ricorso alla ricerca idiografica - nel caso l'intento sia quello di giungere a una comprensione approfondita di una data realtà, considerata nella sua unicità e specificità.

Con l'applicazione del metodo positivo alle scienze umane, dalla fine del secolo XIX, alle precedenti prospettive teoretiche e storiografiche si sono aggiunti gli indirizzi della ricerca "empirica», intesa non già come mera raccolta di fatti, ma come indagine che parte dall'esperienza e torna all'esperienza; tali sono la ricerca descrittiva, con metodo osservativo, e la ricerca sperimentale propriamente detta.

Come è noto, esistono diversi livelli d'indagine empirica: un primo livello, descrittivo, si propone di fornire una rappresentazione accurata e fedele di quanto accade; un ulteriore livello, correlazionale, cerca di stabilire come ciò che accade è in relazione con alcune dimensioni (variabili); infine l'indagine sperimentale si pone l'obiettivo di produrre informazioni circa le relazioni causali.

Le indagini dell'ultimo livello - le verifiche sperimentali - mirano ad indurre delle costanti, a isolare l'azione di uno o più fattori fatti variare nella innovazione, a generalizzare delle conclusioni diverse o supposte ottimali, e simili (Calonghi, 1986, p. 70). Gli esperimenti, infatti, devono consentire dei ragionamenti, delle argomentazioni in base ai quali si possa dire se l'ipotesi formulata è vera o falsa perché sono state rilevate o meno, empiricamente, le conseguenze che si erano previste come derivanti da quella ipotesi all'atto in cui era stata formulata (ivi, p. 86).

Naturalmente i livelli, così come gli obiettivi di una ricerca, non sono quasi mai mutuamente escludenti: infatti le ricerche sono spesso «miste», ovvero prevalentemente "descrittivo-correlazionali» e "correlazionali-sperimentali». Le differenze che si presentano nell'uso dell'uno e dell'altro metodo dipendono dal differente peso che viene dato ai diversi aspetti della realtà.

I metodi quantitativi offrono maggiori possibilità di generalizzazione, sebbene si debba riconoscere che in campo educativo non è mai possibile una generalizzazione assoluta. Il valore dei metodi qualitativi sta nella concreta descrizione dei dettagli del processo stesso nel suo svolgimento.

Coggi (2005, pp. 26-27) afferma che la ricerca qualitativa applicata all'educazione in senso lato ha lo scopo di «comprendere la realtà educativa 
indagata e approfondirne le specificità mediante il coinvolgimento e la partecipazione personale del ricercatore.

La ricerca qualitativa non si vanta di avere messo a punto metodi specifici, ma preferisce piuttosto ricorrere di volta in volta all'analisi semiotica del discorso, del contenuto, della narrazione, e persino all'analisi statistica (Denzin \& Lincoln, 1994). La ricerca qualitativa fa anche uso dei metodi della ricerca naturalistica o etnografica.

L'approccio qualitativo offre alcuni vantaggi rispetto a quello quantitativo: il contatto con la «vita reale», il radicamento nel locale, la ricchezza e la visione organica, la capacità di studiare i processi. Inoltre, si presta in maniera particolarmente efficace allo studio dei «significati». I dati qualitativi infatti sottolineano l'importanza dell'esperienza vissuta e quindi sono particolarmente adatti a individuare i significati che le persone attribuiscono agli eventi, ai processi, e alle strutture della loro vita - percezioni, assunti, pregiudizi e presupposizioni - e a collegare questi significati al mondo che li circonda.

I metodi qualitativi sono efficaci anche in altre tre aree. Permettono di esplorare nuovi campi di ricerca e di sviluppare ipotesi che possono essere successivamente verificate impiegando metodi quantitativi; possono a loro volta verificare delle ipotesi; ma soprattutto sono particolarmente utili quando occorre complementare, convalidare, spiegare, illuminare, o reinterpretare i dati quantitativi raccolti nello stesso ambito (Lumbelli, 1984).

L'epistemologia del nuovo realismo, nella misura in cui ha sviluppato nei ricercatori la consapevolezza dei limiti dell'ermeneutica influenzata dal pensiero debole, ha aperto la strada all'eclettismo metodologico che mira a cogliere l'ampia sfaccettatura della realtà educativa in tutte le sue dimensioni.

\section{TIPOLOGIE DI DISEGNO DI RICERCA CON I METODI MISTI}

L'esame finora svolto sul contributo del realismo («nuovo» o "critico») allo sviluppo della ricerca empirica in campo educativo deve condurre a conclusioni operative che possano orientare il lavoro dei ricercatori. Come si è detto, oggi si preferiscono i metodi misti e ciò influisce nella scelta dei piani o disegni di ricerca. Alla luce di quanto è stato precedentemente esposto, tra le tipologie di disegno di ricerca con i metodi misti tre sembrano offrire maggiori garanzie di scientificità: convergente parallelo, sequenziale esplicativo, sequenziale esplorativo (Creswell \& Plano Clark, 2011).

1. - Il disegno convergente parallelo prevede contemporaneamente l'utilizzo del metodo quantitativo e qualitativo, con la stessa priorità e seguendo le stesse fasi. L'integrazione è prevista unicamente a conclusione dello studio, 
durante l'interpretazione globale dei risultati derivati dall'applicazione parallela dei metodi.

Dopo aver raccolto entrambe le tipologie di dati simultaneamente ma separatamente si procede con l'analisi distinta dei due data base, seguendo le procedure relative ai due differenti approcci di ricerca. I metodi vengono poi integrati attraverso l'unione di risultati, durante la loro interpretazione (e qualche volta durante l'analisi dei dati) ${ }^{7}$.

I propositi di un disegno convergente parallelo possono essere rivolti ad una più completa comprensione delle due fonti di dati o a corroborare i risultati ottenuti da differenti metodi o, ancora, a comparare più livelli d'analisi all'interno di un sistema.

Wittink, Barg e Gallo (2006), ad esempio, lo utilizzano all'interno di uno studio in ambito medico mirato a comprendere la convergenza o meno tra le valutazioni di medici e pazienti rispetto allo status di depressione di questi ultimi. In generale, questo disegno si sceglie se si ha la necessità di raccogliere entrambe le tipologie di dati in un unico momento e quando a queste si attribuisce un uguale valore e priorità per la comprensione del problema di ricerca.

Il disegno richiede una certa expertise nella ricerca sia quantitativa che qualitativa da parte del ricercatore, che può agire individualmente e, forse più efficacemente, con un team. Esso pone essenzialmente delle problematiche legate alla definizione dei campioni ed alla loro dimensione, così come alla difficoltà di far convergere i due insiemi di dati ed alla risoluzione dei casi, peraltro frequenti, in cui i risultati dei due percorsi paralleli sono discrepanti.

2. - Il disegno sequenziale esplicativo prevede la conduzione di una iniziale fase quantitativa e, sui risultati di quest'ultima, un follow up attraverso una seconda fase qualitativa allo scopo di dare una spiegazione più chiara ed approfondita dei primi risultati. Il ricercatore connette le due fasi utilizzando i risultati quantitativi per formulare le domande di ricerca qualitative, impostare il campionamento e la raccolta dei dati.

L'obiettivo è quello spiegare qualitativamente i risultati numerici che necessitano di ulteriore esplorazione, e di utilizzare questi stessi per selezionare intenzionalmente i partecipanti più adatti per lo studio qualitativo.

Questo disegno si usa se il ricercatore ha un orientamento prevalentemente quantitativo o anche se il problema di ricerca presuppone una risposta prettamente quantitativa, come nei casi in cui si ha a disposizione una letteratura sul fenomeno in oggetto, per cui siano già note le variabili chiave e gli strumenti per misurarle.

7 Tramite un processo di trasformazione dei dati, per cui si distingue la quantizzazione dei dati qualitativi o la qualitizzazione dei dati quantitativi. 
In questo caso, nonostante si attribuisca una priorità maggiore al metodo quantitativo, l'approccio qualitativo ha comunque un peso importante nella logica del disegno, dal momento che serve per dare il vero significato ai risultati e trova la sua più adeguata applicazione nei casi in cui, da una prima analisi quantitativa, si evincano dati poco chiari, contradditori rispetto alla letteratura preesistente, sorprendenti o addirittura non significativi.

Aspetto problematico di questo disegno è la previsione, lungo la prima fase, delle strategie che permetteranno di ricavare le informazioni su cui basare la scelta del campione nella successiva fase qualitativa. In altre parole, vi è la necessità di decidere su quali risultati fare il follow up e quali criteri utilizzare per selezionare i partecipanti, se ad esempio, considerare i gruppi che per punteggio si distinguono all'interno del campione quantitativo, o gli outliner per comprenderne il significato, o ancora trarre delle indicazioni per selezionare, al di fuori del campione esaminato, altri soggetti tipici o rappresentativi dei differenti gruppi studiati quantitativamente.

Un classico esempio di disegno sequenziale esplicativo è il lavoro di Ivankova e Stick (2007) finalizzato allo studio dei fattori che influenzano la persistenza allo studio degli studenti nei percorsi di alta formazione come il dottorato ${ }^{8}$.

Nel complesso, il disegno sequenziale esplicativo, pur indirizzandosi verso un approccio emergente, appare semplicemente diretto all'implementazione di due fasi distinte, tanto che anche il Report finale può essere scritto separatamente. Nella pratica le due fasi richiedono molto tempo per l'attuazione; inoltre può essere difficile ottenere l'approvazione per la conduzione di una seconda fase di ricerca, quando non possono essere specificati in fase progettuale i risultati della prima fase.

3. - Il disegno sequenziale esplorativo è costituito sempre da due fasi consecutive e si distingue dal disegno esplicativo poiché, al contrario di questo, parte da una fase qualitativa esplorativa, che ha una priorità maggiore, per poter poi avere quanti più elementi possibili per costruire la fase quantitativa. Le fasi vengono messe in connessione utilizzando i risultati qualitativi per costruire la fase quantitativa, specificando le domande di ricerca e le variabili che la guideranno. Il disegno si propone di esplorare il perché della scarsa conoscenza di teorie e variabili relativamente ad un certo fenomeno, e/o di

8 Gli autori hanno dapprima impostato una fase quantitativa ampia, misurando la persistenza allo studio su un campione di oltre 200 soggetti in fasi diverse dello stesso percorso formativo; successivamente, dall'analisi di questi dati, hanno rilevato l'esistenza di 4 diversi gruppi di soggetti distinti per livelli di persistenza e, all'interno di ciascuno di questi, hanno selezionato un soggetto definibile «tipico» per quel gruppo sulla base della media dei punteggi ottenuti quantitativamente, per poi avviare quattro casi di studio in profondità. 
costruire uno strumento quantitativo o, ancora, di valutare se le tematiche qualitative possono essere generalizzabili ad una popolazione.

Il criterio principale che orienta la ricerca nei vari casi da affrontare, è legato, innanzitutto, all'esperienza e alle attitudini del ricercatore. È evidente poi che la scelta del metodo di indagine è strettamente collegata a quanto si vuole ottenere come risultato.

Nonostante i vantaggi che è facile intravedere nell'utilizzo dei metodi misti, che appaiono più che altro "pratici» agli occhi del ricercatore, il quale può cercare la soluzione dei suoi quesiti utilizzando all'occorrenza dati numerici così come narrativi, in una logica ora induttiva ora deduttiva, va da sé che una loro applicazione scientificamente programmata è tutt'altro che facile e immediata (Bazeley, 2003).

Per utilizzare questo approccio metodologico è essenziale non solo possedere delle buone capacità di ricerca in ambito sia quantitativo che qualitativo, ma soprattutto riflettere bene in fase di progettazione su come si intende integrare e mettere in connessione i due metodi usati ed i rispettivi risultati.

Se questo passaggio non viene chiaramente definito e giustificato a prio$r i$, come è facilmente intuibile, si può incappare in notevoli problemi in fase di interpretazione e lettura dei dati. La conoscenza dei limiti e dei vantaggi di questo nuovo filone di ricerca, a fronte della sua odierna portata, diviene allora uno dei tasselli fondamentali per il necessario continuo sviluppo di consapevolezza nei ricercatori, che sono chiamati a rendere sempre più esplicite le ragioni delle proprie scelte metodologiche.

\section{RIFERIMENTI BIBLIOGRAFICI}

Bazeley, P. (2003). Teaching mixed methods. Qualitative Research Journal, Special Issue, 4, 117-126. http://rosma212.files.wordpress.com/2010/08/bazeley-p2003-teaching-mixed-methods.pdf.

Calonghi, L. (1957). La sperimentazione e i suoi pericoli. Orientamenti Pedagogici, 4, 635-641.

Calonghi, L. (1973). Esperienza, sperimentazione, aggiornamento. Scuola di Base, 20(2-3), 7.

Calonghi, L. (1986). Sperimentazione nella scuola. Roma: Armando.

Calvani, A. (1998). Ricerca qualitativa e costruttivismo: tra vecchie questioni e nuovi paradigmi. Studium Educationis, 2, 231-241.

Coggi, C., \& Ricchiardi, P. (2005). Progettare la ricerca empirica in educazione. Roma: Carocci.

Corallo, G. (1966). Il lavoro scientifico. Fondamenti e metodi. Bari: Adriatica. 
Corallo, G. (2010). Pedagogia, Vol. 1-2. Roma: Armando.

Creswell, J. W., \& Plano Clark, V. L. (2011). Designing and conducting mixed methods research. Thousand Oaks, CA: Sage.

De Caro, M. \& Ferraris, M. (a cura di). (2012). Bentornata realtà. Torino: Einaudi.

Ferraris, M. (2012). Manifesto del nuovo realismo. Roma - Bari: Laterza.

Garcia Hoz, V. (2003). Metodi quantitativi e metodi qualitativi nella ricerca sperimentale in campo educativo. In G. Zanniello (a cura di), La prepedagogicità della sperimentazione (pp. 181-188). Palermo: Palumbo.

Gilson, É. (2008), Il realismo, metodo della filosofia, Roma: Leonardo da Vinci. (ed. orig., Le réalisme méthodique. Paris: Téqui, 1935).

Ivankova, N. V., \& Stick, S. (2007). Students' persistence in a distributed doctoral program in educational leadership in higher education: A mixed methods study. Research in Higher Education, 48(1), 93-135.

Lumbelli, L. (1984). Qualità e quantità nella ricerca empirica in pedagogia. In E. Becchi \& B. Vertecchi (a cura di), Manuale critico della sperimentazione e della ricerca educativa (pp. 101-133). Milano: Franco Angeli.

Mari, G. (2013). Nuovo realismo, vecchi problemi. Pedagogia e Vita, 71, 188-201.

Pellerey, M. (2011). La scelta del metodo di ricerca. Riflessioni orientative. Giornale Italiano della Ricerca Educativa, 4(7), 107-111.

Polanyi, M. (1959). The study of man. Chicago: University of Chicago Press.

Polanyi, M. (1990). La conoscenza personale. Verso una filosofia post-critica. Milano: Rusconi (ed. orig., Personal knowledge. Towards a post-critical philosophy. London: Routledge - Kegan, 1958).

Possenti V. (2013). Realismo diretto e verità. In A. Lavazza \& V. Possenti (a cura di), Perché essere realisti. Una sfida filosofica. Milano: Mimesis.

Rorty, R. (1994). Scritti filosofici, Vol. 1. Roma - Bari: Laterza.

Searle, J. (2012). Prospettive per un nuovo realismo. In De Caro \& Ferraris, 2012 (pp. 167-188).

Torrance, T. F. (1992). Il recupero del realismo nella moderna epistemologia e il pensiero di Michael Polanyi (1984). In Id., Senso del divino e scienza moderna (pp. 193-281). Città del Vaticano: LEV.

Trinchero, R. (2004). I metodi della ricerca educativa. Roma - Bari: Laterza.

Vinti, C. (1999). Michael Polanyi. Conoscenza scientifica e immaginazione creativa. Roma: Studium.

Wittink, M. N., Barg, F., \& Gallo, J. J. (2006). Unwritten rules of talking to doctors about depression: Integrating qualitative and quantitative methods. Annals of Family Medicine, 4, 302-309. 


\section{Riassunto}

Il nuovo realismo ha evidenziato i limiti del costruttivismo sociale e del cosiddetto "pensiero debole", che rischia di sfociare nel nichilismo. Sono stati recentemente rimossi dei pregiudizi che impedivano di cogliere la realtà dei fatti educativi nella sua concreta evidenza. Tuttavia un'apertura alla prospettiva metafisica offrirebbe al nuovo realismo ulteriori spazi epistemologici. In tale senso il contributo del realismo critico potrebbe aiutare a superare l'artificiosa antinomia tra il ruolo attivo del soggetto conoscente e la rilevazione oggettiva dei fatti educativi. Se si realizzasse questa apertura la conoscenza scientifica e la conoscenza corrente non sarebbero più viste come antinomiche perché la seconda costituirebbe un approfondimento della prima, in una linea di continuità. I metodi sperimentali di ricerca in campo educativo si sono recentemente avvalsi delle riflessioni filosofiche dei realisti per integrare l'approccio qualitativo e quello quantitativo allo studio della realtà dell'educazione. Anche i nuovi disegni di ricerca che intendono seguire e rilevare, senza ingabbiarli, $i$ processi educativi sono per lo più misti.

Parole chiave: Approccio qualitativo, Approccio quantitativo, Disegni di ricerca, Metodo sperimentale, Realismo critico. 